\title{
Modeling of Signal Generation Function and Electron Penetration Effects on the EBIC Signal of Schottky diodes of Ge
}

\author{
${ }^{1}$ Beggah Yamina and ${ }^{2}$ Lahreche Abderezzek \\ ${ }^{1}$ Faculty of Science of the Engineer, University of Jijel, \\ Zone Aouled Aissa, Bp 98, 18000 Jijel, Algeria \\ ${ }^{2}$ Faculty of Science, University of Jijel, Zone Aouled Aissa, \\ BP 98, 18000 Jijel, Algeria
}

\begin{abstract}
The present study shows that simple forms for the generation functions, if connected to appropriate electronic path (electron range), can be successfully used to describe the collection efficiency in EBIC devices. Both uniform and point-like source generation functions are considered here and for which two phenomenological electronic paths, depending on the incident beam energy, are obtained. Complexities that might arise solving the continuity equations are, thus, avoided. The calculations were performed for the Schottky diodes $\mathrm{Au} / \mathrm{Ge}$.
\end{abstract}

Key words: Elecdtron beam induced current (EBIC), generation function, electron penetration, schottky diodes of Ge

\section{INTRODUCTION}

The Electron Beam Induced Current (EBIC) is one of the frequently used methods in the Scanning Electron Microscopy (SEM) to investigate the electrical properties of defects in semiconductors ${ }^{[1]}$. The electron beam has, in fact, many advantages compared to other sources of excitations (optical, injection, etc.) since its energy and cross section (which determine the depth and the volume of the excitation) are precisely controllable. The recombination of the carriers at extended defects such as dislocations or grain boundary can be imaged and the EBIC contrast can be measured $^{[2-3]}$. In absence of defects, the collected current is mainly a function of the minority carrier diffusion length which is related to the carrier lifetime. These two parameters can give us some handle on the material quality. There are, however, many factors that influence the measured current, such as carrier diffusion, carrier recombination, the excitation geometry and others which might render the quantitative interpretation of the experimental results somehow difficult. In this context, different approaches have been proposed for the modelling of the induced current and the subsequent determination of the carriers diffusion and recombination parameters following an adequate fit of experimental data.

In all cases, the EBIC current is calculated by solving the continuity equation for the excess carriers which can be expressed for one dimension as:

$$
\frac{d j}{d z}=g(z)-r(z)
$$

Where $\mathrm{g}(\mathrm{z})$ and $\mathrm{r}(\mathrm{z})$ are, respectively, the generation and recombination rate of the electron-hole pairs at the depth $\mathrm{z} . \mathrm{J}$ is the carrier flux.

Simple forms of $g(z)$ such as the punctual generation at a depth $\mathrm{z}_{0}^{\left[{ }^{[6]}\right.}$-which is usually used to calculate the EBIC contrast- or the uniform generation in a sphere ${ }^{[7]}$. The aim of this paper is to show that such simplified expression of $\mathrm{g}(\mathrm{z})$ can be used only if an appropriate electronic path is considered. The dependence of this phenomenological electron range on the beam energy is derived for both uniform and pointlike source generation

\section{THEORY}

General: The last Eq. 1 explicitly involves both the generation and recombination rates of hole-electron pairs at a given depth in the solid material. Of practical interest is, consequently, the knowledge of the energy dissipation profile in a solid as a function of the penetration depth when such a solid is bombarded by an electronic or a photonic beam. It is clear, that the choice of a particular form of $\mathrm{g}(\mathrm{z})$ is of great importance in the $\mathrm{J}(\mathrm{z})$ computation and hence in the collected EBIC currents. Many analytical expressions of $\mathrm{g}(\mathrm{z})$ exist in

Corresponding Author: Beggah Yamina, Faculty of Science of the Engineer, University of Jijel, Zone Aouled Aissa, Bp 98, 18000 Jijel, Algeria 
the literature ${ }^{[4-8]}$. The most used is the modified Gaussian $\mathrm{g}_{\mathrm{G}}$ developed by $\mathrm{Kyser}^{[4]}$ and given by the Eq. 2:

$$
\begin{aligned}
\mathrm{g}_{\mathrm{G}}(\mathrm{z})= & A \exp \left[-\left(\frac{\mathrm{z} / \mathrm{R}_{\mathrm{K}}-\mathrm{u}_{0}}{\Delta \mathrm{u}}\right)^{2}\right] \\
& -\mathrm{B} \exp \left(\frac{-\mathrm{bz} / \mathrm{R}_{\mathrm{K}}}{\mathrm{u}_{0}}\right)
\end{aligned}
$$

$\mathrm{A}, \mathrm{B}, \mathrm{u}_{\mathrm{o}}, \Delta \mathrm{u}$ and $\mathrm{b}$ are constants.

Its adequate range-energy function $\mathrm{R}_{\mathrm{K}}$ developed by Wittry and Kyser ${ }^{[9]}$ is given by the Eq. 3:

$$
\rho R_{K}\left(g / \mathrm{cm}^{2}\right)=2.5610^{-3}\left(E_{0} / 30\right)^{1.68}
$$

Other forms for the energy dissipation (depth-dose) functions have been proposed by other authors. Of particular interest is the polynomial form $\mathrm{g}_{\mathrm{PL}}$ developed by Everhart and $\mathrm{Hoff}^{[5]}$ and given by the Eq. 4:

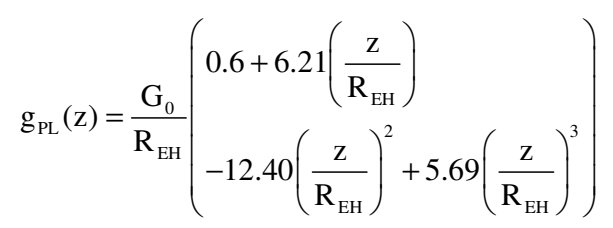

with $\mathrm{G}_{0}$ being the total generation rate.

Its adequate range-energy function $\mathrm{R}_{\mathrm{EH}}$ developed by the same authors is given by the Eq. 5 :

$$
\rho R_{\mathrm{EH}}\left(\mathrm{g} / \mathrm{cm}^{2}\right)=4.010^{-6} \mathrm{E}_{0}^{1.75}
$$

In the Eq. 3 and $5 \rho$ indicates the density of the target; $\mathrm{E}_{\mathrm{o}}$ being the primary beam energy.

In their pioneering work which we will adopt in this work, $\mathrm{Wu}$ and Wittry ${ }^{[10]}(\mathrm{W}-\mathrm{W})$ used a modified approximation (Eq. 2) to the depth distribution of the energy dissipation function as the basis to analytically derive expressions for the induced current and collection efficiency. The latter was used to successfully fit efficiency data obtained from measurement on a number of Schottky barrier devices and, in particular, to quantitatively extract the diffusion lengths of excess carriers and the average energy required to generate a hole-electron pair.

EBIC collection efficiency of schottky contacts theory: In this study we will used the W-W method (planar configuration), which is illustrated in Fig. 1.

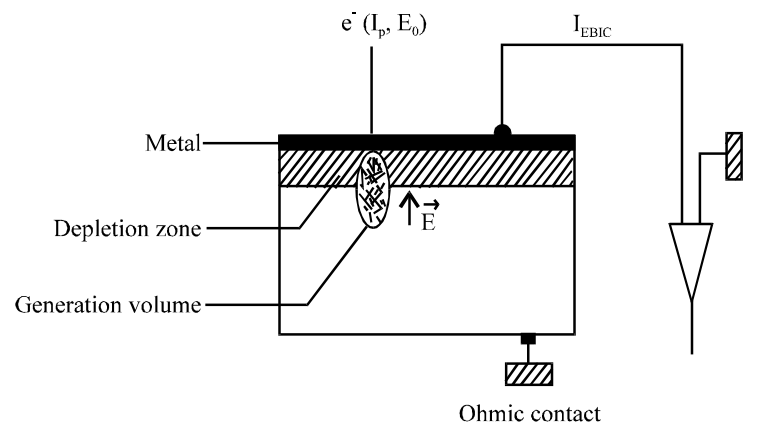

Fig. 1: Geometry of the experimental EBIC technique in the planar junction configuration

The electron beam is exposed to a metalsemiconductor junction. As the beam voltage is raised, the center of the excitation distribution will move deeper into the sample. The excess carriers will diffuse toward the junction, where they are collected. The collected current $I_{c}$ will depend primarily on the range of the generated carriers and the diffusion length of the minority carriers. The theoretical collected current depends on the sum o two currents, $I_{d}$ and $I_{b}$. $I_{d}$ is the current due to carriers generated in the depletion region, while $I_{b}$ is the current due to carriers generated in the bulk of the semiconductor. By assuming a collection probability near unity inside the depletion layer, $\mathrm{Wu}$ and Wittry have derived expressions for $\mathrm{I}_{\mathrm{d}}$ and $\mathrm{I}_{\mathrm{b}}$. The theoretical collection efficiency is defined as the ratio of the collected current $I_{c}=I_{d}+I_{b}$ and the generated one $\mathrm{I}_{\mathrm{g}}$ :

$$
\eta\left(E_{o}\right)=\left(I_{d}+I_{b}\right) / I_{g}
$$

$\mathrm{W}-\mathrm{W}$ model was the first one giving the dependence of the curves $\eta$ on the primary beam energy $E_{0}$.

The theoretical collection efficiency will depend on only four parameters:

$$
\eta=f\left(R, L, z_{m}, w\right)
$$

Where:

$\mathrm{R}=$ The maximum range of electrons

$\mathrm{L}=$ The diffusion length

$\mathrm{z}_{\mathrm{m}}=$ The metal layer thickness and $\mathrm{w}$ the depletion layer width

\section{RESULTS AND DISCUSSION}

The influence of electron range and generation function on collection efficiency: 
The calculations of the curves $\eta\left(E_{0}\right)$ relate to Schottky diodes containing Germanium with a high doping to give a depletion zone of about $\mathrm{w}=0.23 \mu \mathrm{m}$. We choose a low diffusion length $\mathrm{L}=2 \mu \mathrm{m}$ to make readable any change which may appear between the curves especially in the bulk. The metal ( $\mathrm{Au})$ thickness is $\mathrm{z}_{\mathrm{m}}=25 \mathrm{~nm}$.

The collection efficiency curves $\eta\left(\mathrm{E}_{\mathrm{o}}\right)$ are calculated for the modified Gaussian $\mathrm{g}_{\mathrm{G}}$ and the polynomial $\mathrm{g}_{\mathrm{PL}}$ generation forms of carriers which are coupled to their adequate electronic paths. Fig. 2 shows the curve 1 and the curve 2 which are calculated with $\mathrm{g}_{\mathrm{G}}$ and $\mathrm{R}_{\mathrm{K}}$ and with $\mathrm{g}_{\mathrm{PL}}$ and $\mathrm{R}_{\mathrm{EH}}$ respectively. If we interchange, we calculate the curve 3 with $\mathrm{g}_{\mathrm{G}}$ and $\mathrm{R}_{\mathrm{EH}}$ and the curve 4 with $g_{P L}$ and $R_{K}$. It can be seen clearly from Fig. 2 that the difference between the values of the curves 1 and 2 is quite small (less than $1 \%$ at high beam energy). So, the curve 1 and the curve 2 have been taken as reference and this designation will be adopted in the remainder of this paper. The two other curves are quite different from the reference ones.

In Fig. 2 calculation uses a modified Gaussian generation $\mathrm{g}_{\mathrm{G}}$ with Kyser range-energy function of electron $R_{K}$ (curve 1), a polynomial generation $g_{P L}$ with Everhart-Hoff range-energy function of electrons $\mathrm{R}_{\mathrm{EH}}$ (curve2), $\mathrm{g}_{\mathrm{G}}$ with $\mathrm{R}_{\mathrm{EH}}$ (curve3) and $\mathrm{g}_{\mathrm{PL}}$ with $\mathrm{R}_{\mathrm{K}}$ (curve 4). Calculations are carried out using $\mathrm{Wu}$ and Wittry model ${ }^{[2]}$ and the following parameters: depletion zone $=0.23 \mu \mathrm{m}$, metal thickness $=25 \mathrm{~nm}$ and diffusion length $L_{p}=2 \mu \mathrm{m}$.

In the other side, we will consider two simplified generation function. The uniform one $g_{U}$ is expressed in function of u, simply by:

$$
g_{U}(u=z / R)=G_{o}
$$

$\mathrm{u}$ being the carriers penetration normalized to the maximum range $\mathrm{R}$ of electrons.

The punctual generation $\mathrm{g}_{\mathrm{PC}}$ is expressed by:

$$
g_{P C}(u=z / R)=G_{o} \delta(u-1 / 3)
$$

The generation point is situated at $1 / 3$ of the maximum range of electrons $\mathrm{R}$ which was adopted by Watanabe ${ }^{[11]}$.

The two last generation functions are each one coupled to $R_{K}$ and $R_{E H}$ to calculate four curves $\eta\left(E_{o}\right)$ : $g_{U}$ with $R_{K}$ (curve 3 ), $g_{P C}$ with $R_{K}$ (curve 4 ), $g_{U}$ with $R_{E H}$ (curve 5) and $g_{P C}$ with $R_{E H}$ (curve 6). These four curves are reported in Fig. 3 with the references ones, to be compared.

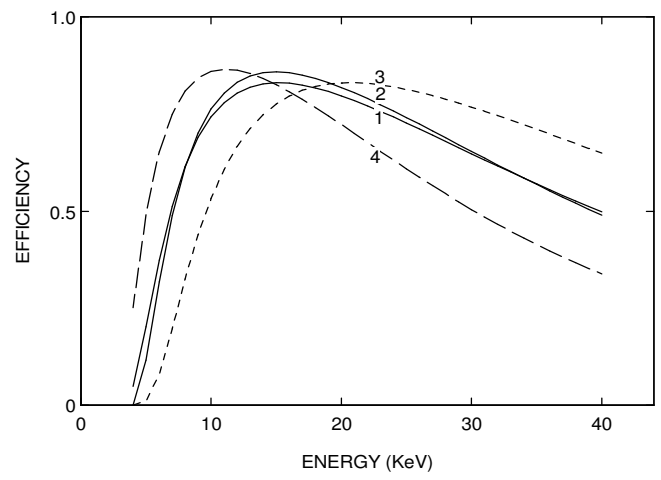

Fig. 2: Collection efficiency curves for an Au/Ge Schottky contact

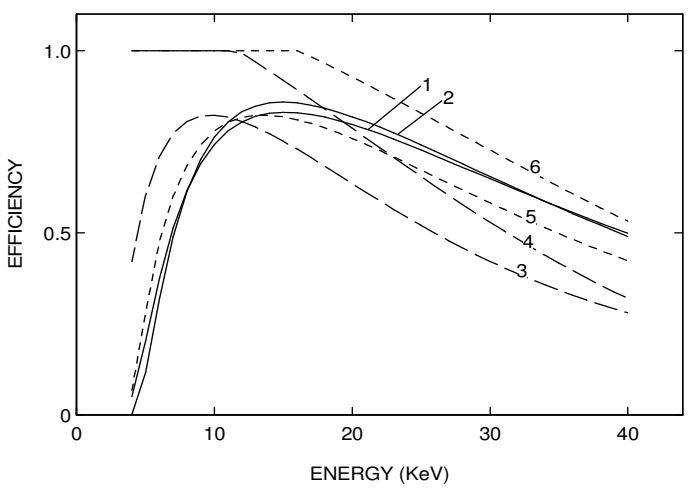

Fig. 3: Collection efficiency curves for an $\mathrm{Au} / \mathrm{Ge}$ Schottky contact

In this figure the calculation uses the same curves 1 and 2 (reference curves) as in Fig. 2 and a uniform generation $g_{U}$ with $R_{K}$ (curve 3), $g_{U}$ with $R_{E H}$ (curve 5), a punctual generation $g_{P C}$ with $R_{K}$ (curve 4) and $g_{P C}$ with $\mathrm{R}_{\mathrm{EH}}$ (curve 6). Calculations are carried out using $\mathrm{Wu}$ and Wittry model ${ }^{[2]}$ and the same parameters as in Fig. 2.

It is shown clearly that the effect of the parameter $\mathrm{R}$ on the collection efficiency is fairly noticeable. In the light of these various observations, it can be conclude that the choice of a given form of the generation function or the electronic path is basic for the analysis of results involving, among others, the determination of some important physical parameters, such as the diffusion length and the recombination speed etc. We can conclude that each form of generation must be coupled with an electronic path specific for it.

On the basis of these conclusions, we construct electronic phenomenological paths for both the uniform generation and for the point-like source one. The values are obtained by an adjusting procedure. Iterations are 
continued, for each given tension of the interval 4-40 $\mathrm{KeV}$, until a value of the ratio $\mathrm{Q}=\left(\eta_{\text {ref }}-\eta_{\mathrm{ph}}\right) / \eta_{\text {ref }}$ less than $2 \%$ is reached, where $\eta_{\text {ref }}$ represents the reference collection efficiency calculated with $\mathrm{g}_{\mathrm{G}}$ and $\mathrm{R}_{\mathrm{K}}$ or with $\mathrm{g}_{\mathrm{PL}}$ and $\mathrm{R}_{\mathrm{EH}}$ (curves 1 and 2 in Fig. 3). But we choose the values $\eta_{\mathrm{ph}}$ which is calculated with the generation functions $g_{U}$ and $g_{P C}$ coupled with $R_{\mathrm{EH}}$ (curves 5 and 6) because they approach the references curves better that those which are calculated with the same generation functions coupled with $R_{K}$ (curves 3 and 4). We will retain the modified value $R_{\mathrm{EH}}$ (in calculating $\eta_{\mathrm{ph}}$ ) which checks the condition mentioned on the ratio $\mathrm{Q}$. All these values which generate the values $\eta_{\mathrm{ph}}$, for the interval of the tensions $\mathrm{E}_{0}$ of use, will be used to rebuilt the electronic phenomenological path from the universal expression of the electron penetration $\mathrm{R}=\mathrm{kE}^{\mathrm{n}}$. In last stage, $\mathrm{k}$ and $\mathrm{n}$ are determined via the least square method. The following expressions have been obtained:

$$
\begin{aligned}
& \mathrm{R}_{\text {phu }}\left(\mathrm{g} / \mathrm{cm}^{2}\right)=5.3010^{-6} \mathrm{E}_{0}^{1.61} \\
& \mathrm{R}_{\text {php }}\left(\mathrm{g} / \mathrm{cm}^{2}\right)=32.910^{-6} \mathrm{E}_{0}^{1.19}
\end{aligned}
$$

$\mathrm{R}_{\text {phu }}$ and $\mathrm{R}_{\text {php }}$ are the electron-range related to the uniform and punctual generation functions, respectively. From our calculation, we find that the point generation is located exactly at $\mathrm{z}_{0}=\mathrm{R}_{\text {php }} / 3$ which has been already adopted by Watanabe ${ }^{[11]}$. Figure 4 reflects the four electron penetration evolution in an Au-nGe Schottky diode: the curve 1 is that calculated by Everhart and Hoff, given by Eq. 5; the curve 3 is that calculated by Kyser, given by Eq. 3 and the curves 2 and 4 are the two electron phenomenological penetrations for the uniform and the point-like source generation functions, given by the Eq. 10 and 11, respectively.

The latter paths have been used for the calculation of the collection efficiency curves. They are shown in Fig. 5 which represent, besides the reference curves already mentioned, those obtained through uniform and point source generation functions, calculated also with $\mathrm{W}-\mathrm{W}$ model ${ }^{[10]}$.

In this last figure calculation uses the same curves 1 and 2 (reference curves) as in Fig. 2 and, a uniform generation $\mathrm{g}_{\mathrm{U}}$ with $\mathrm{R}_{\mathrm{phu}}$ (curve 3) and a punctual generation $g_{P C}$ with $R_{\text {php }}$ (curve 4). Calculations are carried out using Wu and Wittry model ${ }^{[2]}$ and the same parameters as in Fig. 2.

So the curve 3 represents the collection efficiency $\eta_{\text {phu }}\left(E_{0}\right)$ calculated with the uniform

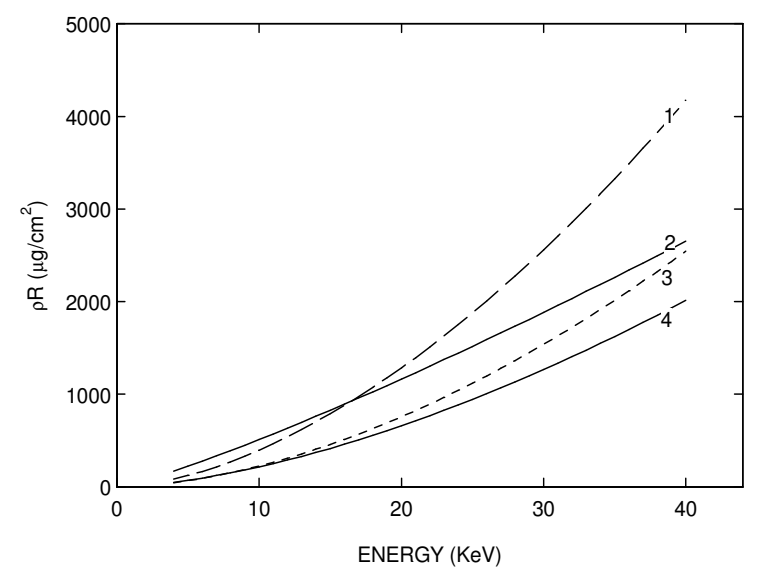

Fig. 4: Optimised electronic path for uniform carrier generation $R_{\text {phu }}$ (curve 2) and punctual carrier generations $R_{\text {php }}$ (curve 4). Curves 1 and 3 correspond to Kyser $\left(\mathrm{R}_{\mathrm{K}}\right)$ and Heverhart-Hoff $\left(\mathrm{R}_{\mathrm{EH}}\right)$ electronic paths, respectively

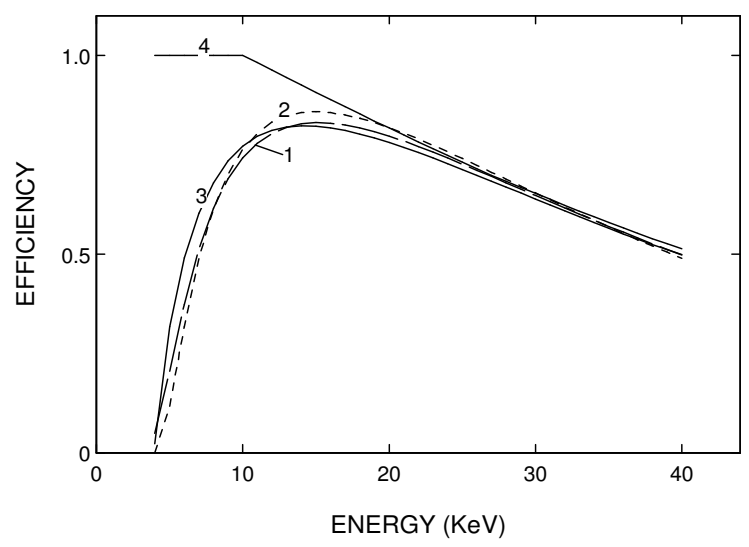

Fig. 5: Collection efficiency curves for an $\mathrm{Au} / \mathrm{Ge}$ Schottky contact

generation function $g_{U}$ coupled with its appropriate electron phenomenological penetration $\mathrm{R}_{\text {phu }}$ given by the Eq. 10 and the curve 4 represents the collection efficiency $\eta_{\text {phu }}\left(\mathrm{E}_{0}\right)$ calculated with the punctual generation function $\mathrm{g}_{\mathrm{PC}}$ coupled with its appropriate electron phenomenological penetration $\mathrm{R}_{\text {php }}$ given by the Eq. 11.

It can be pointed out that our results show clearly that punctual and uniform carrier generation forms can be used if the appropriate electron penetration derived above is considered. Therefore, EBIC contrast dependence on beam energy calculated using such simplified forms must be corrected. 


\section{CONCLUSION}

In the present study, it has been shown that the EBIC signal can be accurately calculated using simple forms of the carrier generation function if an appropriate electron phenomenological penetration $R_{p h}$ is used. $R_{p h}$ dependence on beam energy has been derived for punctual and uniform generation forms which are usually used in order to model EBIC signal. We have shown that it is possible to reach a satisfactory agreement between the collection yield curves thus obtained and those determined on the base of generation and penetration forms more commonly used. It will be interesting to check the method to calculate electron phenomenological path for the case of Schottky diodes of Si.

\section{REFERENCES}

1. Leamy, H.J.,1982. Charge collection scanning electron microscopy. C. J. Applied Phys., 53: R51-R80.

2. Negulyaev, N.N., E.B. Yakimov and S.I. Zaitsev, 2004. Simulation of unchanged dislocation EBIC contrast at high excitation level. Physica Status Solidi, (c), 2 (6): 1822.

3. Chen, J., D. Yang, Z. Xi and A. Sekiguchi, 2005. Recombination activity of Sigma 3 boundaries Influence of iron contamination. J. Applied Phys., 97: 033701.
4. Kyser, D.F., 1972. Electron-Beam-Induced Current in Semiconductors . Proc of the 6th Int. Conf. on $\mathrm{X}$-Rays Optics and Microanalysis-Osaka Univ. of Tokyo Press, pp: 147.

5. Everhart, T.E. and P.H. Hoff, 1971. Determination of Kilovolt Electron Energy Dissipation vs Penetration Distance in Solid Materials. J. Applied Phys., 42 (13) : 5837.

6. Donolato, C., 1978/1979. On the theory of SEM charge-collection imaging of localized defects in semiconductors. Optik, 52: 19.

7. Marek, J., 1982. Scanning electron microscope charge-collection images on grain boundary. J. Applied Phys., 53: 1454.

8. Kanaya, K. and S.J. Okayama, 1972. Electronbeam treated CDO films. Phys. D., 5: 43.

9. Wittry, D.B. and D.F. Kyser, 1967. Measurement of Diffusion lengths in direct-gap semiconductors by electron-beam-induced excitation. J. Applied Phys., 38: 1: 375.

10. Wu, C.J. and D.B. Wittry, 1978. Investigation of minority-carrier diffusion lengths by electron bombardment of Schottky barriers. J. Applied Phys., 49: 2827.

11. Watanabe, M., G. Actor and H.C. Gatos, 1977. Determination of Minority-Carrier lifetime and surface recombination velocity with high special resolution. IEEE Trans. Electron Devises, ED-24: 11-72. 